\title{
Interrelation between the Metabolism of L- Isoleucine and L-Allo-Isoleucine in Patients with Maple Syrup Urine Disease
}

\author{
UDO WENDEL, ULRICH LANGENBECK, AND JAMES W. T. SEAKINS \\ Kinderklinik, Universität Düsseldorf, D-4000 Düsseldorf, [U.W.]; Institut für Humangenetik, Klinikum der \\ Universität Frankfurt-am-Main, D-6000 Frankfurt-am-Main 70, [U.L.], Federal Republic of Germany; \\ Department of Clinical Biochemistry, Institute of Child Health, University of London, \\ London WC1N 1EH, United Kingdom [J.S.]
}

\begin{abstract}
The nonprotein amino acid L-allo-isoleucine is formed endogenously in maple syrup urine disease patients from (R)-3-methyl-2-oxo-pentanoic acid. During strict metabolic balance, the plasma $\mathrm{L}$-allo-isoleucine/Lisoleucine ratio correlates inversely with the residual activity of the branched-chain 2-oxoacid dehydrogenase in fibroblasts and thus constitutes a relevant in vivo parameter of the severity of the metabolic defect in MSUD patients. (Pediatr Res 25:11-14, 1988)
\end{abstract}

\section{Abbreviations}

MSUD, maple syrup urine disease

OMV, oxo-methylvaleric acid

S-OMV (3S)2-oxo-methylvaleric acid, (S)-3-methyl-2-oxopentanoate

R-OMV, (3R)2-oxo-methylvaleric acid, (R)-3-methyl-2oxopentanoate

BCAA, branched-chain amino acids

BCOA, branched-chain 2-oxoacids

In MSUD the oxidative decarboxylation of the branched-chain 2-oxoacids is impaired, and the branched-chain amino acids as well as the corresponding branched-chain 2-oxoacids accumulate in tissues and body fluids. S-OMV, the transamination product of $\mathrm{L}$-isoleucine is a chiral compound. At elevated plasma levels, it has been shown to undergo racemization with formation of $\mathrm{R}$ OMV in vivo (1), apparently via nonenzymic enolization (2).

The metabolic fate of R-OMV is 1 ) transamination to L-alloisoleucine, a non-protein amino acid, regularly found in the patients' blood, and 2), depending on the residual activity of the mutant enzyme, oxidative decarboxylation by which it is channelled into the so-called R-pathway of isoleucine catabolism (3) (Fig. 1).

Sluggish plasma kinetics of allo-isoleucine have been described already by Snyderman and colleagues (4). We observed repeatedly that present allo-isoleucine levels reflect past isoleucine levels and, therefore, aimed at further characterising allo-isoleucine as an inert marker of isoleucine metabolism (5) in patients with classical MSUD and different MSUD variants. In these studies, it turned out that the ratio of allo-isoleucine to isoleucine

Received April 5, 1988; accepted August 18, 1988.

Correspondence Udo Wendel, M.D., Kinderklinik, Universität Düsseldorf Moorenstr. 5, D-4000 Düsseldorf, Federal Republic of Germany.

Supported by Grants We 614/6-2, La 201, and SFB 33 from The Deutsche Forschungsgemeinschaft. concentration under stable metabolic conditions may be used as an in vivo parameter of the severity of the metabolic defect.

\section{MATERIALS AND METHODS}

Plasma BCAA were determined by ion exchange chromatography on automated amino acid analysers (LKB 4400, LKB, Munich; Biotronic 6601, Biotronic, Munich) using the short program for BCAA of Benson et al. (6). Plasma BCOA were determined by gas-chromatrography as the trimethylsilylated quinoxalinole derivates (7). The BCOA decarboxylation activities in the patients' skin fibroblasts were assayed in microtest plates using $\mathrm{L}-\left[1-{ }^{14} \mathrm{C}\right]$ leucine as a substrate $(8)$. Control cell lines $(n=$ 25 ) were from subjects without any metabolic defect and showed a mean activity of $6.18 \pm 2.30 \mathrm{nmol}{ }^{14} \mathrm{CO}_{2} / \mathrm{mg}$ cell protein $/ 3 \mathrm{~h}$ $(\bar{x}+\mathrm{SD})$. Decarboxylation activity of a cell strain at different culture passages was measured with a variation coefficient of about $20 \%$. In most cases of classic MSUD, decarboxylation activity was clearly below $1 \%$ of normal (see Ref. 9 ).

In the present communication, "strict metabolic balance" is defined as constant plasma BCAA levels at a constant BCAA intake during 10 days or more.

\section{PATIENTS}

Six patients with classic and six with variant MSUD were studied. Their classification into variant and classic forms was according to clinical and clinical-chemical findings.

Classic forms. The patients Ca (1980), Öz (1982), Mi (1983), and Ög (1985) presented by the end of the 1st wk of life with pronounced neurologic abnormalities. Diagnosis of MSUD was made between the 11 th and 17 th day of life. At that time, each patient was comatose and had the maple syrup-like odor and a plasma leucine concentration ranging from 2.9-3.7 mmol/liter. So (1981) and En (1975) had a positive family history of classical MSUD, and diagnosis, therefore, was made soon after birth. All patients showed a normal neurologic and somatic development on a BCAA-restricted diet. The tolerance for leucine ranged from $45-55 \mathrm{mg} / \mathrm{kg}$ body weight at the age of $1 \mathrm{yr}$ and from 30 to 35 $\mathrm{mg} / \mathrm{kg}$ at the age of $3 \mathrm{yr}$. Each patient underwent numerous episodes of metabolic derangements triggered by intercurrent illnesses.

Variant forms. Gu (1974) developed neurologic signs at $3 \mathrm{wk}$ of age when plasma leucine concentration was $1.3 \mathrm{mmol} / \mathrm{liter}$. Mo (1977) showed neurologic signs and maple syrup odor on the 10th day of life; diagnosis of MSUD was not made until 2 months of age when plasma leucine concentration was 1.1 $\mathrm{mmol} /$ liter. Both patients showed normal neurologic and somatic development on a BCAA-restricted diet. Leucine tolerance 


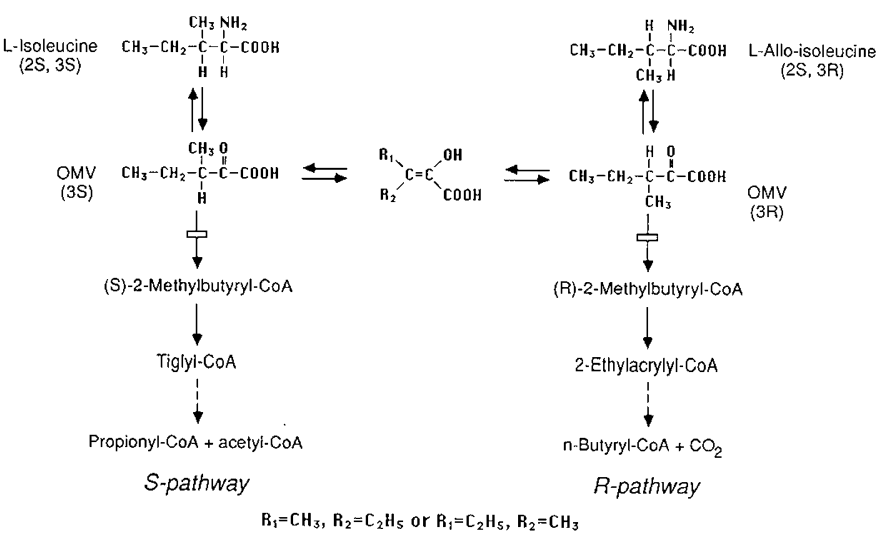

Fig. 1. Catabolism and keto-enol tautomerization of isoleucine and allo-isoleucine. Modified from References 3 and 25.

at the age of $1 \mathrm{yr}$ was about $50 \mathrm{mg} / \mathrm{kg}$ body weight and $40 \mathrm{mg} /$ $\mathrm{kg}$ at the age of $3 \mathrm{yr}$. They showed only a few episodes of metabolic derangements during intercurrent illnesses.

Ck (1986) was diagnosed as having MSUD at 21 months of age when plasma leucine concentration was $0.8 \mathrm{mmol} /$ liter. During the 1st yr of life, he showed an adverse reaction to protein-rich food. On a protein-restricted diet with $1.4 \mathrm{~g} / \mathrm{kg}$ body weight, the plasma leucine level persisted at about $0.4 \mathrm{mmol} /$ liter during the first 5 months of treatment.

Te (1977) presented with neurologic signs and maple syrup odor at $3 \mathrm{wk}$ of age; she developed normally on a proteinrestricted diet. Febrile illnesses were well tolerated. At a protein intake of $1.3 \mathrm{~g} / \mathrm{kg}$ body weight/day, plasma BCAA levels were only slightly elevated $(2 \times$ normal $)$ during childhood.

In Ku (1979) and $\mathrm{Na}$ (1978), MSUD was detected by neonatal screening. Both patients developed normally on a slightly protein-restricted diet. They never had episodes of metabolic derangements, and plasma leucine levels never exceeded 0.35 $\mathrm{mmol} /$ liter. On $2 \mathrm{~g}$ protein $/ \mathrm{kg}$ body weight, plasma BCAA levels were only slightly $(2 \times$ normal $)$ elevated.

All classical and severe variant patients were maintained on diets sufficiently depleted in the BCAA to keep their plasma concentrations less than $0.5 \mathrm{mmol} /$ liter. In all patients, except for Ög and Ck, the plasma allo-isoleucine/isoleucine ratios were determined at different occasions during the first $5 \mathrm{yr}$ of life at strict metabolic balance.

\section{RESULTS}

Slow plasma kinetics of L-allo-isoleucine can be observed in MSUD patients on extended monitoring as well as after acute Lisoleucine loads. Figure 2 shows the plasma concentrations of the four branched-chain amino acids in a classical MSUD patient on dietotherapy. Whereas L-isoleucine-like L-leucine and Lvaline-oscillated frequently and with high amplitudes, alterations of L-allo-isoleucine followed those of isoleucine with a shift of phase and with a diminished amplitude. This time course is representative of many other similar observations.

An oral L-isoleucine load $(1.5 \mathrm{mmol} / \mathrm{kg}$ body weight $)$ given to a variant MSUD patient produced a rapid rise of the plasma isoleucine concentration (Fig. 3). The peak plasma concentration of OMV followed with a delay of $30 \mathrm{~min}$. Allo-isoleucine appeared in plasma only $3 \mathrm{~h}$ later and plateaued at $0.03 \mathrm{mmol} /$ liter $4 \mathrm{~h}$ after the load. Isoleucine and OMV were eliminated from plasma with a half life of about $3.5 \mathrm{~h}$. Allo-isoleucine remained stable for at least $20 \mathrm{~h}$ and was later cleared from the plasma with a half-life of about $12 \mathrm{~h}$.

Closer scrutinity of our data revealed that, irrespective of possible erratic fluctuations of plasma levels (see Fig. 2), each patient approached a characteristic ratio of allo-isoleucine to isoleucine levels at periods of strict metabolic balance. The ratio, in the individual patients, did not change with a consistent trend during the study periods of 2 to $5 \mathrm{yr}$. These ratios of plasma concentrations are given in Table 1 for the different subjects with MSUD. In addition, their residual BCOA dehydrogenase activities are shown as measured in cultured skin fibroblasts.

In classic MSUD patients, the ratio ranged from 0.6-0.75. In the group comprising the different MSUD variants $(\mathrm{Gu}, \mathrm{Mo}, \mathrm{Ck}$, $\mathrm{Te}, \mathrm{Ku}, \mathrm{Na}$ ), the ratios were lower than in classic MSUD and were inversely related to the residual BCOA dehydrogenase activity. In very mild MSUD variants, (with $15-20 \%$ residual enzyme activity), the ratio was 0.1 . No relationship was found between this ratio and the plasma concentration of the other BCAA for the patients listed in Table 1 .

Under strict metabolic balance, allo-isoleucine plasma concentrations were always lower than plasma isoleucine concentrations. However, when isoleucine intake was acutely diminished or when protein synthesis abruptly increased, plasma isoleucine levels fell below those of allo-isoleucine. This happened frequently in classic MSUD (see Fig. 2). In very mild variants we did not find any condition in which plasma allo-isoleucine concentration exceeded that of isoleucine.

\section{DISCUSSION}

The nonprotein amino acid L-allo-isoleucine is an endogenous, regular, and specific constituent of plasma in MSUD patients (10). From its L-configuration (11) it follows that it is formed from R-OMV and that the equilibrium of plasma isoleucine and allo-isoleucine in metabolically stable MSUD patients occurs by racemization of the chiral OMV through keto-enol tautomerism. This reaction proceeds nonenzymatically $(2,12)$ in an as yet unknown compartment and is easily observed, e.g. at very high concentrations of the isoleucine-derived S-OMV (13). Weinberg and Walser (13) described a polarimetric assay of both OMV dinitrophenylhydrazone enantiomers. The original procedure requires large sample volumes and has not yet been adapted to studies in humans. A potentially more sensitive separation of the diastereomeric quinoxalinol propionic acid derivatives of S- and R-OMV could not be achieved on a great number of gaschromatographic columns, including Chirasil-Val (Langenbeck U 1986, unpublished data). Until now, any interpretation of in vivo metabolism of allo-isoleucine in patients could be based only indirectly on plasma levels of allo-isoleucine alone, and theoretically also on measurements of R-pathway metabolites, which are diminished in concentration in MSUD urine compared to normal controls (3).

The slow plasma kinetics of allo-isoleucine in MSUD, which were initially reported by Snyderman (4) and which are highlighted again in our studies, just accentuate the normal state: Whereas the initial plasma half-life of $\mathrm{L}$-isoleucine after an oral load in normal subjects is $45-70 \mathrm{~min}(14,15)$, L-allo-isoleucine has a plasma half-life of a few hours (4). In classic MSUD, the respective values are about 2 days for $\mathrm{L}$-isoleucine and between 5.5-7.5 days for L-allo-isoleucine (4). Interestingly, allo-isoleucine does not support rat growth (16), whereas its keto-analogue, R-OMV, does $(16,17)$.

These observations suggest that, in reference subjects, transamination of L-allo-isoleucine to R-OMV is slow and that any $\mathrm{R}-\mathrm{OMV}$ formed is channeled preferentially into the R-pathway. Only high intracellular concentrations of R-OMV would saturate the R-pathway and allow formation of isoleucine via racemization to S-OMV. Recent biochemical studies in normal human fibroblasts (18) have confirmed the first prediction, demonstrating that transamination of allo-isoleucine is only $41 \%$ of the rate of isoleucine.

In classic MSUD, the R-pathway is closed to R-OMV catabolism, and allo-isoleucine can be cleared by formation of isoleucine and by (inefficient) renal excretion. Both OMV enantiomers appear to be present in MSUD patients, because the percentage atom excess of ${ }^{15} \mathrm{~N}$ of isoleucine and allo-isoleucine could not be 

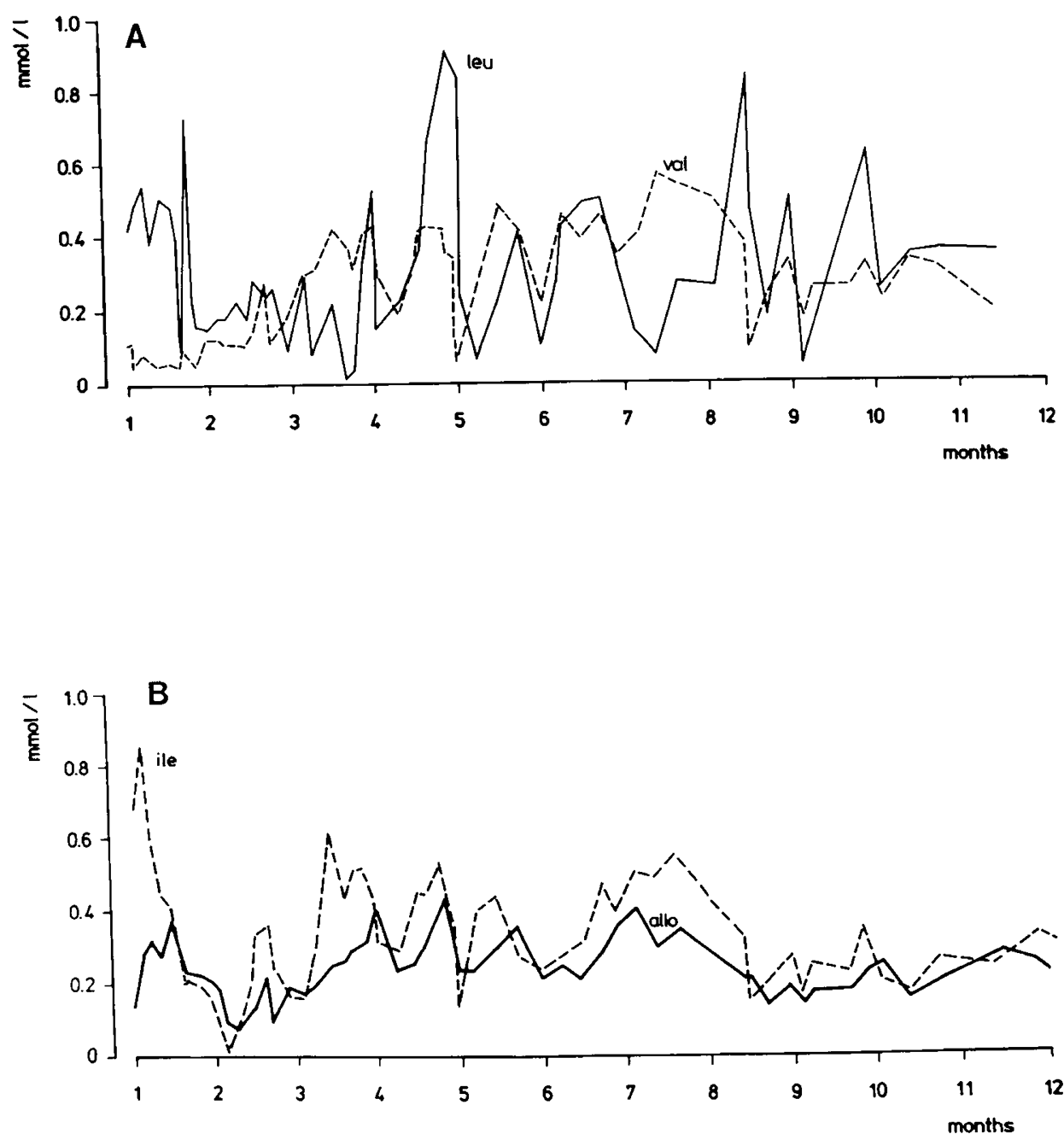

Fig. 2. Plasma concentrations of the four BCAA L-leucine $(l e u)$ and L-valine $(v a l)(A)$, and L-isoleucine (ile), and L-allo-isoleucine $($ allo $)(B)$ in a classic MSUD female (So) on dietotherapy in her 1st yr of life.

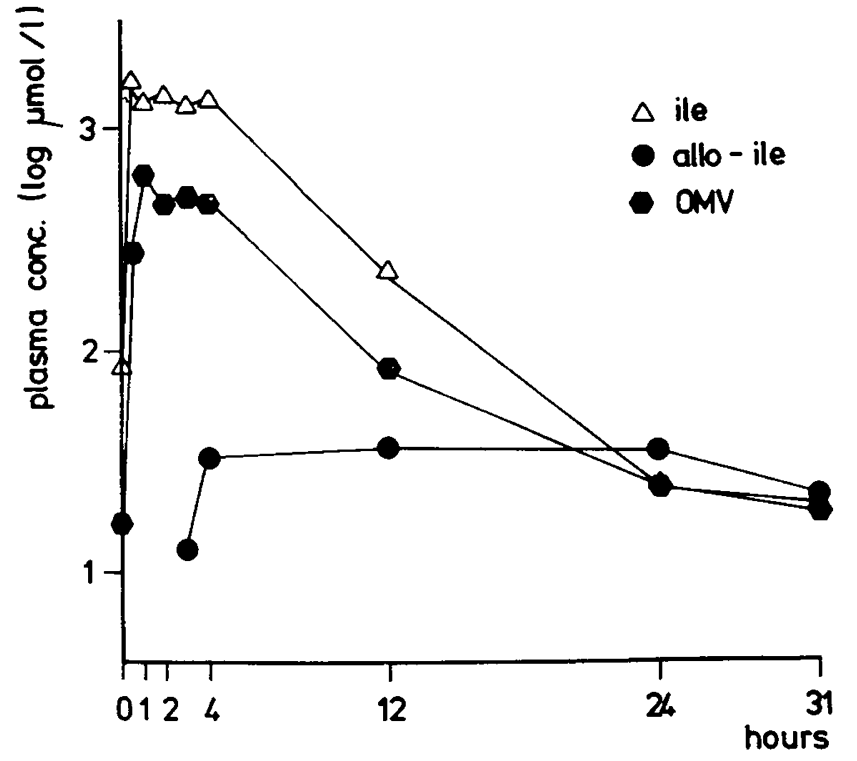

Fig. 3. Time course of plasma concentrations of L-isoleucine (ile), Lallo-isoleucine (allo-ile), and OMV after an oral load of L-isoleucine (1.5 $\mathrm{mmol} / \mathrm{kg}$ body weight) to a patient with mild MSUD variant (Te; $13 \%$ residual BCOA dehydrogenase activity in fibroblasts). The plasma concentrations are given after $\log 10$ transformation of the data.
Table 1. Plasma allo-ile/ile concentration ratios in MSUD subjects during periods of strict metabolic balance*

\begin{tabular}{|c|c|c|c|c|}
\hline Course & Patient & $\begin{array}{l}\text { Mean allo-ile/ile } \\
\text { ratio } \pm \mathrm{SD}\end{array}$ & $(n) \dagger$ & $\begin{array}{l}\text { Residual BCOA- } \\
\text { dehydrogenase } \\
\text { activity }(\%)\end{array}$ \\
\hline \multirow[t]{6}{*}{ Classic } & (Ög) & $0.70 \pm 0.12$ & $(50)$ & \multirow{6}{*}{$\leqq 1$} \\
\hline & (So) & $0.72 \pm 0.12$ & (108) & \\
\hline & (Ca) & $0.69 \pm 0.13$ & (110) & \\
\hline & (Mi) & $0.67 \pm 0.12$ & $(50)$ & \\
\hline & (Öz) & $0.63 \pm 0.09$ & (106) & \\
\hline & (En) & $0.59 \pm 0.11$ & $(120)$ & \\
\hline \multirow[t]{3}{*}{ Variant } & $(\mathrm{Gu})$ & $0.56 \pm 0.12$ & (104) & 2 \\
\hline & (Mo) & $0.38 \pm 0.07$ & $(63)$ & 3 \\
\hline & (Ck) & $0.27 \pm 0.05$ & (15) & 7 \\
\hline \multirow[t]{3}{*}{ Mild variant } & $(\mathrm{Te})$ & $0.13 \pm 0.03$ & $(22)$ & 13 \\
\hline & $(\mathrm{Ku})$ & $0.12 \pm 0.04$ & (18) & 17 \\
\hline & $(\mathrm{Na})$ & $0.09 \pm 0.04$ & (30) & 19 \\
\hline
\end{tabular}

* $\mathrm{BCOA}$ dehydrogenase activity was assessed in intact cultured fibroblasts with $\left[1-{ }^{14} \mathrm{C}\right]$ leucine $(8)$ in four sets of assays. The plasma isoleucine concentrations ranged from $0.1-0.35 \mathrm{mmol} / \mathrm{liter}$ in variant MSUD subjects and from $0.1-0.55$ in classic MSUD subjects. Taking the Spearman rank correlation as test criterion, the inverse relation of both parameters is statistically highly significant $(p<1 \%)$.

$\dagger n$, number of determinations. 
distinguished 60 to $240 \mathrm{~min}$ after a ${ }^{15} \mathrm{~N}$-leucine load (19), whereas de novo formation of allo-isoleucine from isoleucine is much slower (20) (Fig. 3). We anticipate from these data that racemization of OMV is also slow in MSUD, and that under high intracellular concentrations of the BCOA retransamination of allo-isoleucine to R-OMV is even slower than in controls. Testing such a "trap model" of allo-isoleucine metabolism in MSUD will require in vitro studies on the mutual transamination of alloisoleucine with the BCOA. The reportedly very low concentrations of 2-hydroxy-3-methylpentanoic acid in MSUD (21) make relevant contributions of this compound to allo-isoleucine kinetics unlikely.

The supposed allo-isoleucine trap would become more leaky with increasing residual BCOA dehydrogenase activities in MSUD variants, because the transamination equilibrium of alloisoleucine would be shifted to R-OMV by partial R-OMV catabolism. Our data in Table 1 indicate that by this chain of events plasma half-life of allo-isoleucine will depend in MSUD variants on the residual activity of the BCOA dehydrogenase complex. By L-allo-isoleucine loading tests in selected MSUD patients, we have preliminarily proved this prediction (22).

For many investigators, leucine tolerance is thought to be a better predictor of clinical severity in MSUD than in vitro enzyme data (see citations in Ref. 23). Indirectly probing the residual in vivo capacity of the R-pathway of allo-isoleucine as shown in our report may further improve clinical evaluation of variant MSUD patients, including the assessment of thiamine responsiveness (24) and estimation of the necessary long-term dietary prognosis. The practice of adjusting acute dietary needs through monitoring of leucine levels is not impeded, of course, by these considerations.

\section{REFERENCES}

1. Walser M, Sapir DG, Mitch WE, Chan W 1981 Effects of branched-chain ketoacids in normal subjects and patients. In: Walser M, Williamson JR (eds) Metabolic and clinical implications of branched-chain amino and ketoacids. Elsevier/North Holland, New York, pp 291-299

2. Meister A 1951 Studies of d-and $1-\alpha$-keto- $\beta$-methylvaleric acid. J Biol Chem 190:269-276

3. Mamer OA, Tjoa SS, Scriver CR, Klassen GA 1976 Demonstration of a new mammalian isoleucine catabolic pathway yielding an $\mathrm{R}$ series of metabolites. Biochem J 160:417-426

4. Snyderman SE, Norton PM, Roitman E, Holt LE 1964 Maple syrup urine disease, with particular reference to dietotherapy. Pediatrics 34:454-472

5. Wendel U, Langenbeck U, Luthe H, Seakins WT 1986 L-allo-isoleucine-an inert marker of isoleucine metabolism in maple syrup urine disease. Eur $J$ Pediatr 144:523

6. Benson JV, Cormick J, Patterson JA 1967 Accelerated chromatography of amino acids associated with phenylketonuria, leucinosis (maple syrup urine disease), and other inborn errors of metabolism. Ann Biochem 18:481-492
7. Langenbeck U, Wendel U, Mench-Hoinowski A, Kuschel D, Becker K, Przyrembel $\mathrm{H}$, Bremer HJ 1978 Correlations between branched-chain amino acids and branched-chain $\alpha$-keto acids in blood in maple syrup urine disease. Clin Chin Acta 88:283-291

8. Wendel U, Gamm G, Claussen U 1981 Maple syrup urine disease: $\alpha$-ketoisocaproate decarboxylation activity in different types of cultures amniotic fluid cells. Prenatal Diagn 1:235-240

9. Dancis J, Hutzler J, Snyderman SE, Cox RP 1972 Enzyme activity in classical and variant forms of maple syrup urine disease. J Pediatr 81:312-320

10. Norton PM, Roitman E, Snyderman SE, Holt LE 1962 New findings in maple syrup urine disease. Lancet 1:26-27

11. Halpern B, Pollock GE 1970 The configuration of alloisoleucine present in maple syrup urine disease. Biochem Med 4:352-356

12. Snyderman SE 1967 Maple-syrup-urine disease. In: Nyhan WL (ed) Amino Acid Metabolism and Genetic Variation. McGraw Hill, New York, pp 171183

13. Weinberg RB, Walser M 1977 Racemization and amination of the keto-analog of isoleucine in the intact dog. Biochem Med 17:164-172

14. Koch-Schweitzer H 1984 Über den Einfluß einer oralen Gabe verzweigtkettiger Ketosäuren und Aminosäuren auf die Konzentrationen dieser Substanzen im Liquor cerebrospinalis beim Menschen. Medical Dissertation, University of Göttingen

15. Kuschel D 1979 Das Verhalten der verzweigtkettigen Ketosäuren im Blut nach peroraler Leucinbelastung: ein Beitrag zur Frage der Interaktionen im Stoffwechsel der verzweigtkettigen Aminosäuren. Medical Dissertation University of Göttingen

16. Meister A, White J 1961 Growth response of the rat to the keto analogs of leucine and isoleucine. J Biol Chem 191:211-216

17. Funk MA, Lowry KR, Baker DH 1987 Utilization of the L- and DL-isomers of $\alpha$-keto- $\beta$-methylvaleric acid by rats and comparative efficacy of the keto analogs of branched-chain amino acids provided as ornithine, lysine and histidine salts. J Nutr 117:1550-1555

18. Schadewaldt P, Wendel U 1987 Comparison of the catabolism of branchedchain L-amino acids in cultured human skin fibroblasts. Pediatr Res 22:591594

19. Matthews DE, Ben-Galim E, Haymond MW, Bier DM 1980 Allo-isoleucine formation in maple syrup urine disease: isotopic evidence for the mechanism. Pediatr Res 14:854-857

20. Wendel U, Lombeck I, Bremer HJ 1983 Maple syrup urine disease. N Engl J Med 308:1100-1101

21. Shigematsu Y, Kikuchi K, Momoi T, Sudo M, Kikawa Y, Nosaka K, Kuriyama M, Haruki S, Sanada K, Hamano N, Suzuki Y 1983 Organic acids and branched-chain amino acids in body fluids before and after multiple exchange transfusions in maple syrup urine disease. J Inherited Metab Dis 6:183-189

22. Wendel U, Schadewaldt P, Langenbeck U 1987 Metabolism of L-isoleucine and L-allo-isoleucine in patients with maple syrup urine disease in healthy subjects. Abstracts of the 25th SSIEM Annual Symposium, p 47

23. Langenbeck U 1984 Pathobiochemical and pathophysiologic analysis of the MSUD phenotype. In: Adibi SA, Fekl W, Langenbeck U, Schauder P (eds) Branched-Chain Amino and Ketoacids in Health and Disease. Karger, Basel, pp 315-334

24. Fernhoff PM, Lubitz D, Danner DJ, Dembure PP, Schwartz HP, Hillman R, Bier DM, Elsas LJ 1985 Thiamine response in maple syrup urine disease. Pediatr Res 19:1011-1016

25. Tanaka K, Rosenberg LE 1983 Disorders of branched-chain amino acid and organic acid metabolism. In: Stanbury JB, Wyngaarden JB, Fredrickson DS, Goldstein JL, Brown MS (eds) The Metabolic Basis of Inherited Disease, 5th ed. McGraw-Hill, New York, pp 440-473 